\title{
A Three-Phase Open Hardware Design for Power Quality Solutions
}

\author{
Francisco G. Montoya ${ }^{1}$, Alfredo Alcayde ${ }^{1}$, Eduardo Viciana ${ }^{1}$, Francisco M. Arrabal-Campos ${ }^{1}$, Raúl \\ Baños and Juan Martínez-Lao ${ }^{1}$ \\ ${ }^{1}$ Department of Engineering \\ E.S.I., Almería University \\ Carretera de sacramento s/n - La Cañada, 04120 Almería (Spain) \\ Phone/Fax number: +34950214501, e-mail: pagilm@ual.es, aalcayde@ual.es, eviciana@zred.es, fmarrabal@ual.es, \\ rbanos@ual.es,jml357@ual.es
}

\begin{abstract}
The complexity of power grids in terms of generation and consumption transforms the traditional power system to a distributed one. This new paradigm obliges to a better monitoring and control. Herein, we present a low cost and compact device that allows to measure, energy, voltages and currents in three-phase networks. In addition, it has been designed to analyse PQ disturbances. This device is a smart meter and can be considered internet-based power quality monitoring. The statistics data are processed locally with built-in custom software, specifically designed for this purpose, which provides energy consumption or generation patterns in multiple visualization views. The design is released as open hardware and therefore it is presented to the community as a powerful tool. The system was installed in a real photovoltaic power plant over a long time, showing interesting results and findings, which validates our proposal.
\end{abstract}

Key words. Three phase IoT; low cost smart meter; network analyser; electricity; power quality; energy metering.

\section{Introduction}

In 1974, Paraskevakos [1] presented the first remote device for metering electricity. At the same time, the electricity distribution grids have evolved, modernizing and growing. Nevertheless, remote and smart metering were concepts not considered among engineers and scientists for many years. Lately, a lot of issues have arisen, like climate change, trends in the electricity markets, efficiency, and the promotion of renewable energy resources. Moreover, other players in power systems are promoting distributed grids with distributed storage. This new paradigm requires an update in the actual electricity model [2]. The fundamentals of smart grid take into account electricity models and decentralisation in electricity generation. In order to manage these grids, new measurement systems are needed. These systems are usually referred to as smart meters. The European Parliament in its 2012/27/EC directive [3] defines these kind of devices as "an electronic system that can measure energy consumption, providing more information than a conventional meter, and can transmit and receive data using a form of electronic communication."

Nowadays, to know the real state of the power network is one of the biggest challenges that engineers face off. The global trend establishes that power generation systems should be environmentally friendly. This transforms the concept of power generation due to diverse generation points have to supply different consumption points. This transformation yield to a concept called smart grid that allows the delivery the electricity in a controlled environment.

The technology that is able to control this smart grid is based in three principles: control systems, computer processing and advanced renewable energy source [4]. This technology is used to control the increased complexity of networks and in this context arise the power quality (PQ) concept [5]. Millions and millions of electronic devices are connected in combination with distributed microgeneration by renewable energy introducing many disturbances [6]. Some of the PQ disturbances that can be measured in these types of networks are swell, sag, interruption, oscillatory transients, flicker, and harmonic distortion. With the advent of renewable energies, this type of problem will increase due to power electronics. Reactive power, redistribution costs and load reduction costs impose penalties on consumer. In addition, these PQ problems can introduce second-order effects on transformers, incorrect operation of capacitor banks and affect the electronics of sensitive equipment [7]. Solving these problems are the challenges to be addressed in the future for power systems.

Corrective actions to cancel these disturbances must be implemented and executed in the network. The first step is to detect them. Many techniques have been proposed like FFT, S-transform, Hilbert-Huang transform or Wavelet transform. The second step is to recognize them. 
Recognition is based on non-linear optimization techniques. Finally, classify them by means, for example, of neural networks or support vector machines $[8,9]$.

Herein, we propose a new three-phase smart meter which has interesting features like small size, low-cost, precise, and reliable power and electric energy meter. This smart meter allows to visualize energy consumption and has been designed to comply with international standards such as IEC 61000-4-30 and EN-50160. This smart meter is called three phase openZmeter ( $3 \mathrm{p}-\mathrm{oZm}$ from now on). The device is derived as a major improvement from single phase smart meter openZmeter [10]. This device is easily installed in electrical cabinets with a user-friendly supervisory control and data acquisition (SCADA) system. It can be considered an internet-based device; therefore, the device is accessible from anywhere connected to the internet. The interface is simple and intuitive, so people without technical knowledge or skills can easily understand.

The usage of statistics data is organized in an affordable and structured way. The software provides energy consumption or generation patterns in its multiple implemented visualizations. The power required and the energy consumed or generated can be analysed together in such a way that the user can determine, for example, whether they are consuming more or less power than that it is expected.

\section{Three-phase smart meter}

This smart meter can be considered an internet-based power quality monitoring for the three-phase currents and voltages in real time. Currents are sensed by means of external current probes (Rogowski, clamp-on, etc) allowing high-accuracy and high-magnitude currents. The main component is an analog front end (AFE) driven by an STM32F042, which belongs to the STM32 microcontroller family. It has high performance, real-time track signal processing and low-power consumption. The current and voltage signals, converted by means of 12-bit Analog Digital Converter (ADC), are pre-processed by the Digital Signal Processing (DSP) module of the STM32. Complex calculation such as FFT, zero crossing, and RMS values are obtained by an external ARM Linux core.

Figure 1 shows a block diagram of the $3 p-o Z m$ hardware. There are four conceptual blocks. The block for signal acquisition is highlighted in green, the block power supply and management elements in orange, the black block is the STM32 microcontroller, and finally, the yellow block is an advanced RISC machine (ARM) Linux board controller.

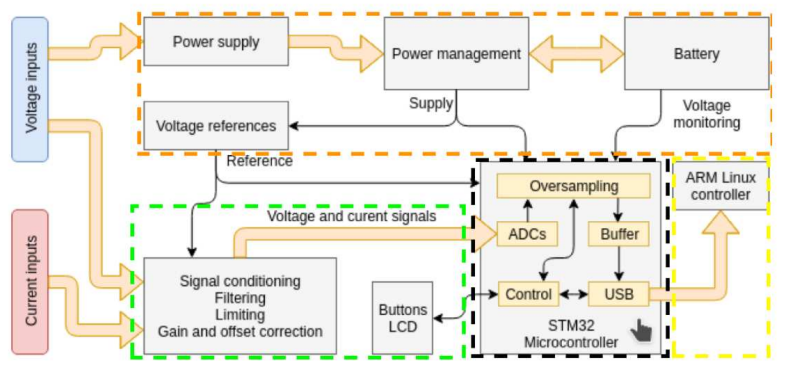

Fig. 1. Three-phase openZmeter (oZm) hardware block diagram.

$3 p-o Z m$ is a compact electronic device. In figure 2, external appearance is shown. The width and height ratio are the typical for a DIN electrical device. The electronic elements are protected with voltage limiter and a fuse for high voltage and high current, respectively.

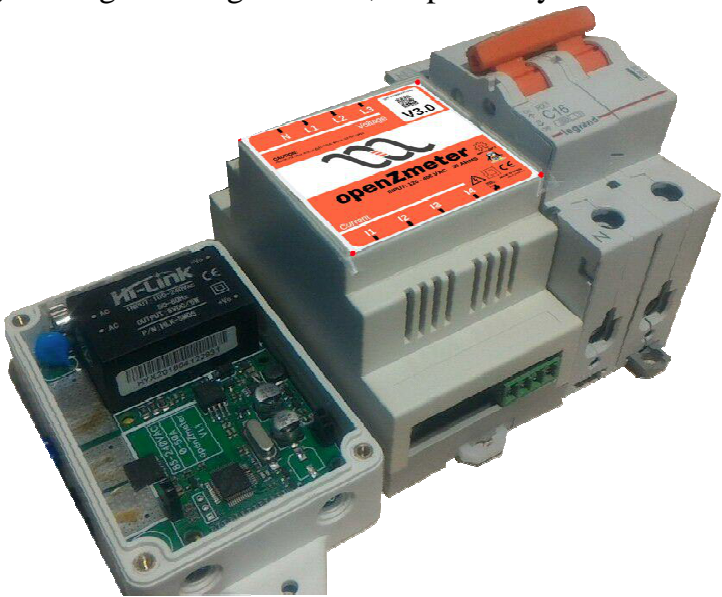

Fig. 2. 3p-oZm full system including plastic enclosure. Size of the device is compared with oZm-1p (left) and two-pole switch (right)

The figure 3 shows the electronic scheme. There are two types of input signals, i.e., voltage signals and current signals. To get the three voltages, the input is filtered and adapted with the MCP6004 operational amplifier and connected to three ADC channels of the STM32. The current is measured using external current probes and similar operational amplifier connected to another four ADC channels of the STM32.

The 3p-oZm software consists of an embedded ARM unit, where a daemon service runs in an endless loop. The ARM board runs a real-time kernel (Linux RT). The daemon service is mainly coded in $\mathrm{C}++$ to obtain a robust and reliable system. As stated before, the device is classified as an Internet-based sensor, therefore it incorporates a wireless module (Ampak AP6212) with WiFi: $802.11 \mathrm{~b} / \mathrm{g} / \mathrm{n}$ and Bluetooth: 4.0 dual mode. In this way, it is possible to connect to the device and monitor the PQ parameters using a web browser with https secure encryption layer.

The real-time electric measurement list is as follows:

- $\quad$ RMS values for voltage and current.

- Active power, reactive power, and apparent power.

- Phase angle for current and voltage. 
- Power factor.

- Harmonics up to 50th for current and voltage.

- $\quad$ Active energy and reactive energy.

- Frequency.

- Voltage events related with PQ disturbances (swell, sags/dips, and interruptions).

The gathered data in the above list of measurements, is presented in nice visualizations with the following features:

- Energy consumption and generation in four quadrants.

- Operation in accordance with international standards IEC 61000-4-30 and EN-50160.

- Aggregation for the voltage channel of $3 \mathrm{~s}, 1 \mathrm{~min}$, $10 \mathrm{~min}$, and $1 \mathrm{~h}$ as extra aggregation for energy metering purposes.

- Alert system and event management (ITIC/CBEMA, frequency, etc.).

- Cutting-edge HTML, Javascript, and CSS3 technologies for a user-friendly dashboard interface.

- API for third-party integration using JSON
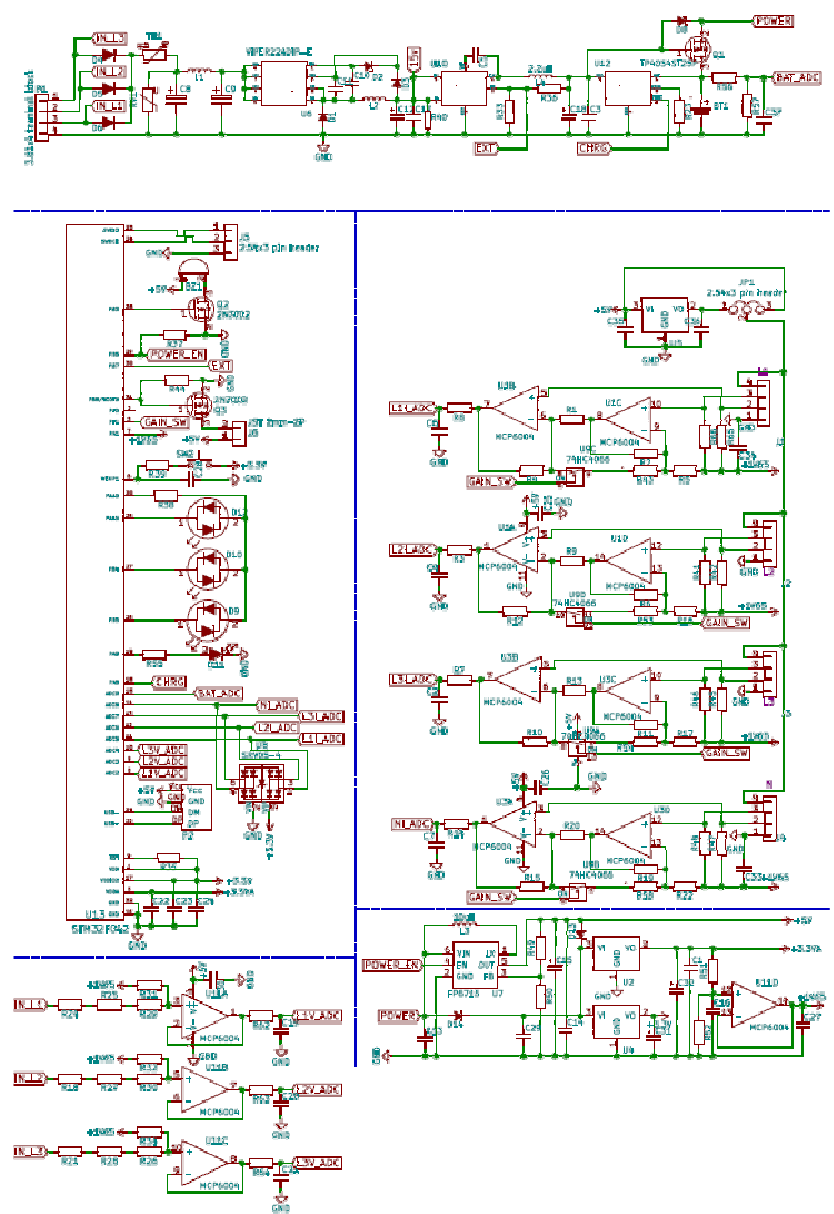

Fig. 3. Electronic scheme of three-phase device.

The installation in a real electric cabinet is very easy. The $3 \mathrm{p}-\mathrm{oZm}$ is designed to be coupled directly to the terminal block. Its placement and wiring are like any electric energy device. The final setup of the $3 \mathrm{p}-\mathrm{oZm}$ installed is shown in the figure 4 . The $3 \mathrm{p}-\mathrm{oZm}$ software setup is configured using whether Ethernet or Wi-Fi or Bluetooth.
The dashboard of 3p-oZm includes the basic magnitudes. The main magnitude is the active energy consumption (kWh), which can be displayed for different periods with different time span aggregation. All the plots shown share a common template based on HTML5, CSS3, JavaScript, and JQuery, thus providing information about the RMS voltage, RMS current, frequency, and active power for a $3 \mathrm{~s}$ aggregation interval. The active energy consumption or generated is shown for a fixed time span, but these data can be aggregated based on nominal values: $3 \mathrm{~s}, 1 \mathrm{~min}, 10 \mathrm{~min}$, and $1 \mathrm{~h}$. The data are stored locally in an SQL-like database (PostgreSQL) and can be retrieved for a day, week, month, or year.

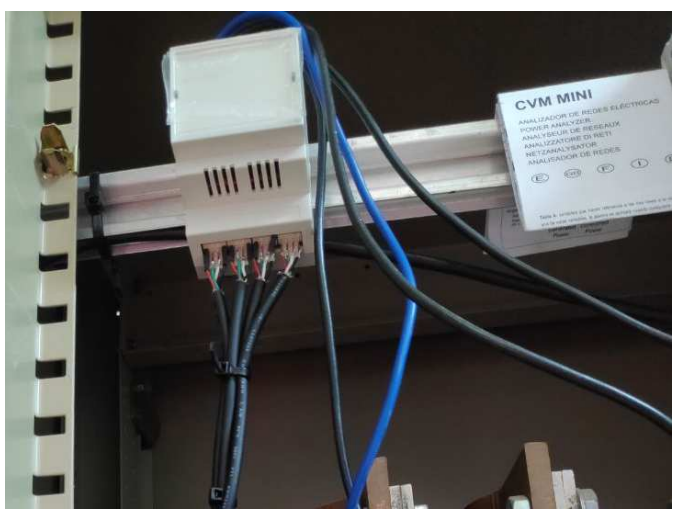

Fig. 4. 3p-oZm installed in a real electric cabinet. The device is fitted to the terminal block like other common electrical devices.

The main signal processing technique implemented in the $3 \mathrm{p}-\mathrm{oZm}$ allows to compute the fast Fourier transform (FFT), in order to retrieve harmonics up to $50^{\text {th }}$ order. As well, it is possible to calculate phasors and detect events of different nature among others features. The figure 5 shows a detailed view of raw samples for voltage and current.

\section{3p-oZm usage in a real photovoltaic power plant}

The photovoltaic power plant is located in Canjayar town (Almería, south of Spain), which is installed on the roof of an industrial building. The photovoltaic power plant connected to the electricity grid, described here, is subject to RD 436/2004, which establishes the premiums for each $\mathrm{kWh}$ injected.

The photovoltaic installation is executed on the roof itself with a $10^{\circ}$ inclination. The installation is oriented $25^{\circ}$ southeast. To avoid shadows, a distance of $0.62 \mathrm{~m}$ is set between rows of modules. The total roof area is 1,015 $\mathrm{m}^{2}$.

Annual energy production is estimated at a total of $146059 \mathrm{kWh}$. The installation has the following technical characteristics:

- $\quad$ Rated power: $100.8 \mathrm{~kW}$.

- Operating voltage: $400 \mathrm{~V}$. 
- $\quad$ Serial/parallel modules: 15/32.

- Orientation: $25^{\circ} \mathrm{W}$.

- Tilt: $10^{\circ}$.

- Solar module: Suntechnics STM 210FWS.

- Inverter: Coenergy- IPG 100K

- Protection and energy panel: circuit breaker, differential, fuses and connection boxes.

$3 \mathrm{p}-\mathrm{oZm}$ has been installed in this photovoltaic power plant. The installation was done in an easy way in the electric cabinet. In the figure 5 , it is shown the real installation in the protection panel inside of the cabinet.

All data captured are displayed in real time and they are presented using an intuitive dashboard, where the data are refreshed dynamically using cutting-edge web technologies. The dashboard is accessible from the internet through the following url: https://openzmeter.com/myozm.

The dashboard is web friendly and responsive for usage devices like mobiles and tablets. The figure 6 shows two captures of the access to the $3 \mathrm{p}$-oZm using a commercial mobile phone. Main parameters measured and other parameters like device state are shown in the top of the view. It is possible to check the current, voltage and the energy generation using tactile displacement. Also, the access to the data and all features is possible anywhere and anytime.

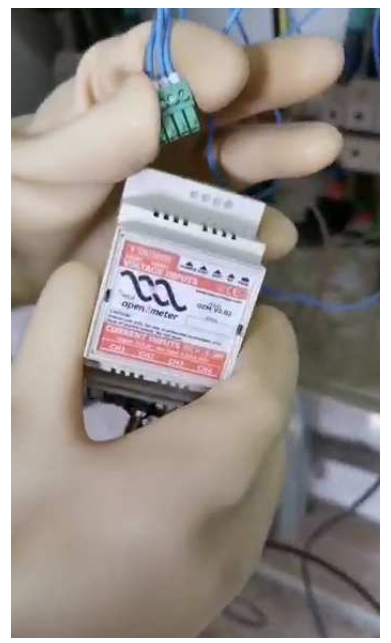

Fig. 5. Easy installation in the cabinet of the photovoltaic power plant. The blue wire corresponds to those used for the voltage sensor; the connector is designed for an easy installation in one click.
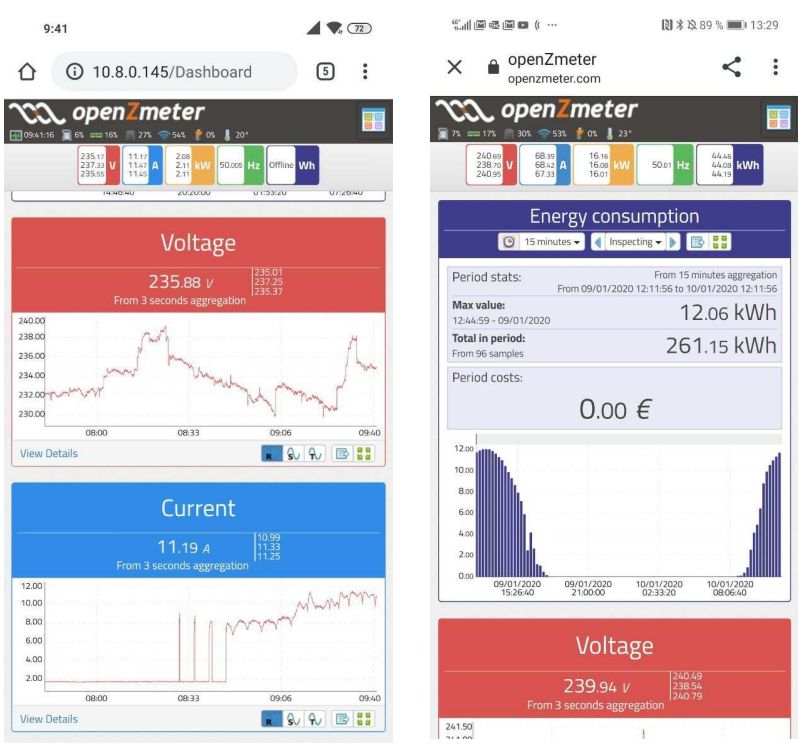

Fig. 6. Display of the three voltage and current phases and the different options for advanced signal processing techniques available in the $3 \mathrm{p}-\mathrm{oZm}$. In the top, the user can observe the main magnitude of the photovoltaic power plant.

The main view is divided in five blocks, which are plotted for active energy consumption, RMS voltage, RMS current, active power, and grid frequency. Each plot can be maximized for better inspection.

All blocks are plotted using a $3 \mathrm{~s}$ aggregation interval over the previous $2 \mathrm{~h}$ as default option, to obtain finegrained resolution. Moreover, the energy block has a more advanced features for choosing other resolutions or aggregations. They are synchronized to display information for the same time frame. The rest of the web interface is divided into six sections: voltage, current, power, frequency, voltage events, and energy stats.

Data collected is shown in figures 7 and 8 accessed using web browser. The information extended is easily presented, where the energy, in this case, is generated in the photovoltaic power plant. As well, it is possible to check the power quality analysis using the options in the side toolbar. This toolbar has different selectable options. First option is the dashboard. The second option is used to analyze data of voltage, current and energy. The third option shows the energy patterns, called active and reactive energy stats. Finally, there are other options related with the configuration device.

An active and reactive energy measurement is carried out by adding the power measurement over time. In the energy stats is possible to get a representation based on heat maps. Each colored "pixel" represents the generation made in a specific time span that can be adjusted between $10 \mathrm{~min}$ and $1 \mathrm{~h}$ so that there is resolution according to the user's interests. For a better analysis, two visualizations were implemented oriented to tables, where it is possible to get data table depending on the year, month, week of the year, or day of the week. 
Energy generation patterns can be easily detected. In figure 8 , the power generation plot shows, for example, when the power plant is working. For a photovoltaic power plant only generates from dawn till dusk. The typical generation with gaussian form is displayed, indicating the correct working of the power plant. As well, it is easy to check when the weather is bad or when it is a cloudy day. The red circle shows when the power plant has generated low energy due to the bad weather.

Data are first retrieved and analyzed using statistical tools, and the results are then displayed in the graphical interface. Each plot can be analyzed with more detail. The main statistical data are average, maximum, and minimum magnitudes of voltage in a time-period using different aggregation scales, and the voltage waveform (3p-oZm can be used as oscilloscope for waveform diagnosis). Plots includes in these views (energy consumption, voltage and current waveforms, frequency, and power quality events) can be easily interpreted by expert and non-expert users. 


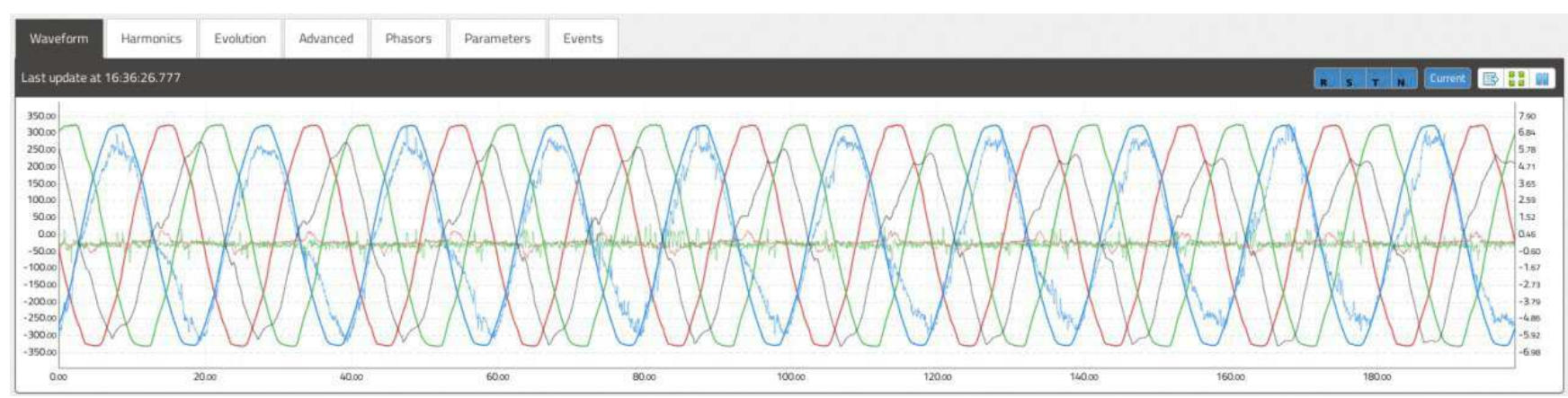

Fig. 8. Display of the three voltage and current phases and the different options for advanced signal processing techniques available in the $3 \mathrm{p}-\mathrm{oZm}$.

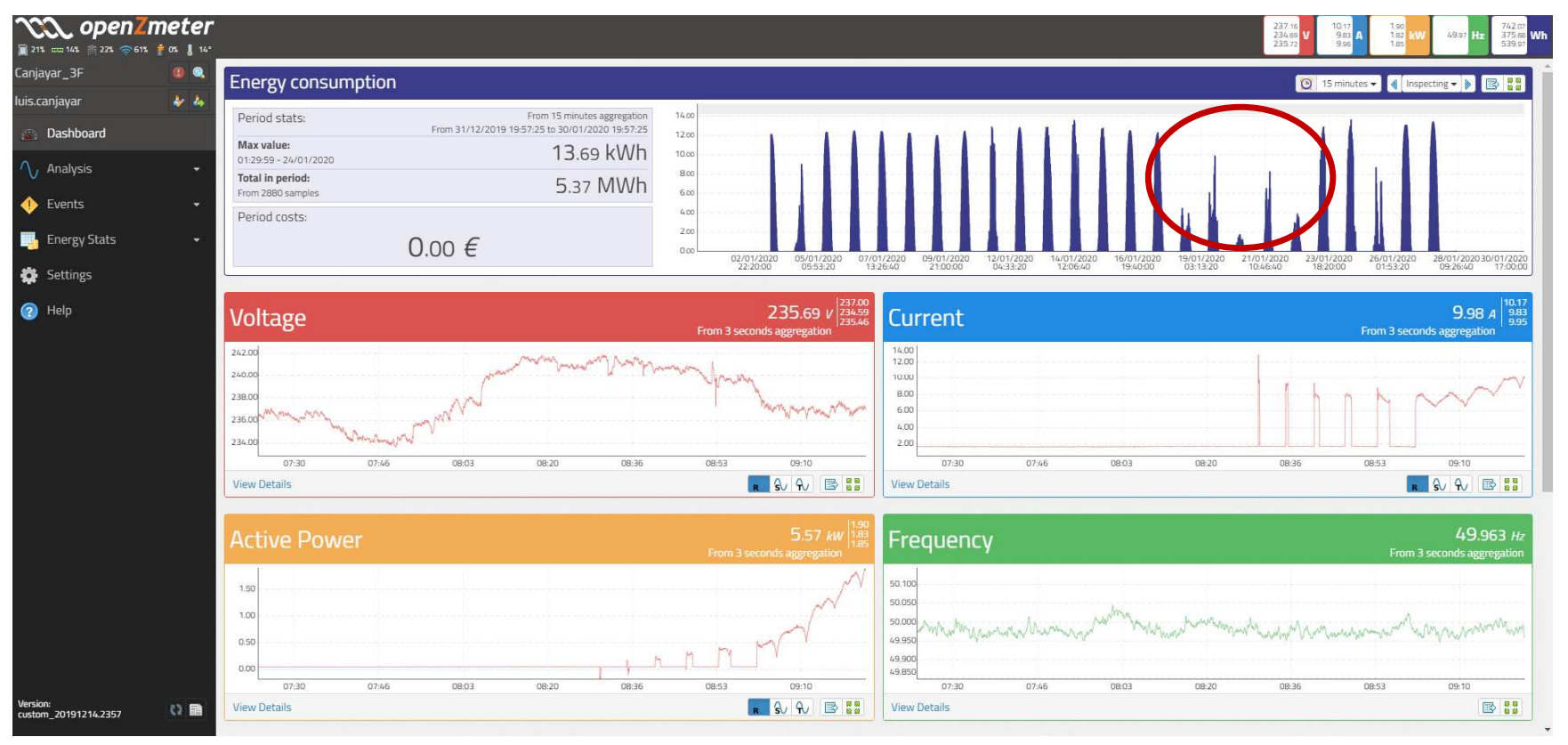

Fig. 9. Energy patterns can be easily detected. The power generation plot shows the pattern of the generation. It is facile to observe when the weather is bad or when it is a cloudy day, the red circle indicates non-sunny days.

\section{Conclusion}

In this paper we present a three-phase smart meter openZmeter (3p-oZm). This device is a major evolution of the single-phase openZmeter. The advanced design of $3 p-$ oZm allows the installation in any cabinet. The algorithm implementation allows high performance, which ensures reliable and accurate energy measurement with the ability to analyse PQ disturbances for the three phases. The user interface is very intuitive and friendly. This device is designed to comply the standards IEC 61000-4-30 and EN-50160. As example, a real photovoltaic power plant located in Canjayar town (Almería, south of Spain) has been monitored installing a $3 \mathrm{p}-\mathrm{oZm}$ in its cabinet. The power plant is installed on the roof of an industrial building. The dashboard is accessible from the internet through the following url: https://openzmeter.com/ myozm. The dashboard is responsive, and the parameter of power generation can be accessible anywhere and anytime.

\section{Acknowledgement}

This research has been supported by the Ministry of Science, Innovation and Universities at the University of Almeria under the programme" Proyectos de I+D de Generación de Conocimiento" of the national programme for the generation of scientific and technological

knowledge and strengthening of the $\mathrm{R}+\mathrm{D}+\mathrm{I}$ system with grant number PGC2018-098813- B-C33.

\section{References}

[1] Paraskevakos, T. Sensor Monitoring Device. U.S. Patent 3,842,208, 15 October 1974

[2] Uribe-Pérez, N.; Hernández, L.; de la Vega, D.; Angulo, I. State of the Art and Trends Review of Smart Metering in Electricity Grids. Appl. Sci. 2016, 6, 68.

[3] Union, E. Directive 2012/27/EU of the European Parliament and of the Council of 25 October 2012 on Energy Efficiency, Amending Directives 2009/125/EC 
and 2010/30/EU and Repealing Directives 2004/8/EC and 2006/32/EC. Available online: https://eurlex.europa.eu/LexUriServ/LexUriServ.do?uri= OJ:L:2012:315: 0001:0056:en:PDF (accessed on 12 January 2019).

[4] C.C. Chu, H.H.C. Iu, Complex networks theory for modern smart grid applications: A survey. IEEE J. Emerg. Sel. Top. Circuits Syst. 2017, 7, 177-191.

[5] International Electrotechnical Commission. Electromagnetic Compatibility (EMC)_Part 4: Testing and Measurement Techniques-Power Quality Measurement Methods; International Electrotechnical Commission: Geneva, Switzerland, 2003; Volume 30.

[6] Y. Shen, F. Hussain, H. Liu, D. Addis, Power quality disturbances classification based on curvelet transform. Int. J. Comput. Appl. 2017, 40, 1-10.

[7] J. Niitsoo, M. Jarkovoi, P. Taklaja, J. Klüss, I. Palu, Power Quality Issues Concerning Photovoltaic Generation in Distribution Grids. Smart Grid Renew. Energy 2015. 6, $148-163$.

[8] Y. Wan, J. Cao, H. Zhang, Z. Zhu, S. Yao, Optimization of the power quality monitor number in Smart Grid. In Proceedings of the 2014 IEEE International Conference on Smart Grid Communications (SmartGridComm), Venice, Italy, 3-6 November 2014, 230-235.

[9] Mohibullah, S.H. Laskar, Power quality issues and need of intelligent PQ monitoring in the smart grid environment. In Proceedings of the 2012 47th International Universities Power Engineering Conference (UPEC), London, UK, 4-7 September 2012, 1-6.

[10] E. Viciana, A. Alcayde, F.G. Montoya, R. Baños, F.M. Arrabal-Campos, F. Manzano-Agugliaro, An Open Hardware Design for Internet of Things Power Quality and Energy Saving Solutions. Sensors 2019, 19, 627. 